\title{
Anaesthesia and Rehabilitative Considerations for Full Mouth Rehabilitation of Cerebral Palsy Patients: A Case Series
}

\author{
Dempsy Chengappa MM ${ }^{1}$, Vidhu Bhatnagar ${ }^{2 *}$, MS Honwad ${ }^{3}$ and Akshai Kannan ${ }^{4}$ \\ ${ }^{1}$ MDS (Paedodontics \& Preventive Dentistry), Oic Dental Center, INHS Sanjivani, Naval Base, Kochi 682004, Kerala \\ ${ }^{2}$ DM (Neuro Anaesthesia), HOD, Department of Anaesthesiology and Critical Care, INHS Sanjivani, Naval Base, Kochi 682004, Kerala \\ ${ }^{3}$ MD (Anaesthesia), CO, INHS Sanjivani, Naval Base, Kochi 682004, Kerala \\ ${ }^{4}$ MDS (Orthodontics \& Dentofacial Orthopaedics), Gd Spl (Orthodontics), Dental Center, INHS Sanjivani, Naval Base, Kochi 682004, Kerala
}

*Corresponding author: Vidhu Bhatnagar, Department of Anaesthesiology and Critical Care, INHS Sanjivani, Naval Base, Kochi 682004, Kerala.

Received Date: December 10, 2020

Published Date: January 05, 2021

\begin{abstract}
Cerebral Palsy (CP) is a central nervous system disorder which is caused due to damage to the developing brain usually occurring in perinatal period. There could be multifactorial etiology with various factors such as maldevelopment of the brain, perinatal stroke, prenatal infection, genetic disorder, ischemic insult in utero or prematurity, thrombophilic disorders, teratogenic exposures, multiple gestations ,maternal fever, exposure to toxins, abdominal trauma, malformation of brain structures, abnormal fetal presentation, instrument delivery, hyperbilirubinemia, meningitis, chronic lung disease, shaken baby syndrome as the possible causes. The children inflicted with $\mathrm{CP}$ are at a higher risk for developing oral manifestations such as Early Childhood Caries (ECC) due to poor oral hygiene, intake of soft diet, difficulty in chewing and swallowing and increased use of sugary oral medications. Behavioural challenges due to associated mental retardation demands pharmacological management under General Anaesthesia (GA) and we present a case series of successful management of Severe ECC in five children diagnosed with CP managed with full mouth rehabilitation under GA. The aim is to highlight importance of counselling, preoperative preparation of patients, surgical modalities and postoperative rehabilitation.
\end{abstract}

Keywords: Intellectual disability; Dental caries; Tooth, Deciduous; Rehabilitation; Central Nervous System Diseases

\section{Introduction}

Cerebral Palsy (CP) is a brain disorder which is caused due to damage to the developing brain usually occurring before or shortly after birth. It is neither progressive nor communicable and causes defects in the central nervous system leading to abnormal motor function, muscle tone and movements [1]. The etiology can be attributed to risk factors which manifest during perinatal period [2]. It has been attributed to multifactorial etiology with various factors such as maldevelopment of the brain, perinatal stroke, prenatal infection, genetic disorder, ischemic insult in utero or prematurity, thrombophilic disorders, teratogenic exposures, multiple gestations, maternal fever, exposure to toxins, abdominal trauma, malformation of brain structures, abnormal fetal presentation, instrument delivery, hyperbilirubinemia, meningitis, chronic lung disease, shaken baby syndrome etc. as its possible causes $[3,4]$. This condition has been classified into various types, most commonly into three major categories which are pyramidal (Spastic), Extrapyramidal and Mixed [5]. The children inflicted with $\mathrm{CP}$ are at a higher risk to develop oral manifestations such dental caries and plaque accumulation as compared to general population due to a variety of factors which result in poor oral hygiene, 
their dietary patterns such as soft diet, difficulty in chewing and swallowing and increased use of oral medications also contribute to the higher risk [6].

There is also an increased incidence of various oral conditions such as gingival hypertrophy, hypoplastic teeth and injuries to upper front teeth in these children [7]. Associated behavioural challenges and the extent of oral manifestations necessitates pharmacological management under General Anaesthesia (GA) to manage defects due to Early Childhood Caries (ECC) $[8,9]$. The administration of GA has inherent risks, and these children are at an increased risk to develop perioperative hypothermia, hypotension, delayed emergence from general anaesthesia, seizures, and airway-related morbidity [10]. We present a case series of successful management of Severe ECC in children diagnosed with CP managed with full mouth rehabilitation under GA with an aim to highlight the various treatment modalities, GA approach and counseling of parents and guardian involved in full mouth rehabilitation of children with ECC requiring full mouth rehabilitation. These children require a multidisciplinary approach involving the Pediatrician, Pediatric Dentist and Anaesthesiologist for effective delivery of care.

\section{Case Report1}

A 5-year-old male child, a diagnosed case of spastic type CP was brought with the complaint of pain in the upper and lower back tooth region for the past 6 months. History of child having difficulty in chewing food and the child appeared malnourished. Patient was not responsive to verbal commands and was undergoing treatment and physiotherapy for CP. The patient was taken to another center for dental treatment earlier, but treatment could not be accomplished due to behavioral issues of the child. The post-natal history of the child revealed a delayed birth cry and subsequent intubation and admission in NICU for seven days. On examination; Intra Oral Examination revealed dental caries in relation to $51,52,5$ $3,54,55,61,62,64,65,74,75,84$ and 85 , pulpal involvement was found in relation to $52,54,62,64,74,84,85$ and 75 was found to be grossly destructed. However, airway examination could not be carried out as patient was not responding to verbal commands. The child was positioned on his parent to provide restraint and the mouth was stabilized using mouth gags to perform the intra oral examination. A provisional diagnosis of chronic irreversible pulpitis in relation to $54,52,62,64,74,75,84,85$, dental caries 51,54,61,64 and grossly destructed 75 was made (Figure 1 ( $a$ and $b$ )). The vital parameters appeared normal however auscultation of chest revealed crackles in basal regions bilaterally.

The treatment plan was devised for complete rehabilitation which included preparatory phase in which the parents were counseled, and dietary instructions were given, a corrective phase which included endodontic treatment and restoration of all restorable teeth and a surgical phase which included extraction of unsavable tooth was drawn out. This treatment plan was discussed with the parents and informed consent was obtained from the parents and Pre anaesthetic checkup (PAC) was performed.

\section{Treatment protocol}

In the surgical phase patient was intubated nasally and pulpectomy was carried out on 54,52,62, 64,74, 84 and 85 , composite restorations were carried out on $55,52,51,61,62,65,84$ and stainless-steel crowns were placed on $54,74,85$. The grossly destructed 75 was extracted after injection of $2 \%$ lignocaine adrenaline 1:80,000 (Figure 1 ( $c$ and d)). Hemostasis was achieved; and preventive care was administered using $2 \%$ sodium fluoride gel. The perioperative period was uneventful and extubation on table performed after removing the throat pack and patient monitored in Post Anaesthesia Care Unit (PACU) with the parents and later shifted to ward. The details of GA approach are discussed separately for all the patients together.

\section{Case Report2}

A 4-year-old male child, a diagnosed case of CP was brought by his parents with complaints of signs of pain and difficulty in chewing food by the child. History by parents revealed premature delivery at 34 weeks of gestation and low birth weight of $1900 \mathrm{gms}$. The child had delayed milestones and was on a semisolid diet (high on carbohydrates and sucrose) prepared in a mixer which could be the major cause behind Severe ECC. On examination; Intra Oral Examination revealed extensive damage to the deciduous dentition, dental Caries with pulpal involvement in relation to $51,52,53,54,61,62,63,64,74,85$. The 84 was grossly destructed and Dental Caries not involving the pulp was seen in relation to 73,83 (Figure 2 ( $\mathrm{a}$ and b)). The lower left deciduous second molars i.e 75 had not erupted fully in the oral cavity and corelated to the finding of delayed milestones. Radiographic investigation in the form of Radiovisiograph (RVG) was carried out with the help of parental help to restrain the child and this confirmed the final diagnosis (Figure 2e).

A treatment plan was devised, parents were counseled, and dietary instructions were given in preparatory phase, corrective phase which included endodontic treatment and restoration of all restorable teeth and a surgical phase which included extraction of unsavable tooth was drawn out. Informed consent was obtained from the parents and PAC was carried out. In the surgical phase patient was orally intubated and throat pack. The child was scrubbed and draped; oral hygiene measures were carried out using a toothbrush. Pulpectomy was carried out on $51,52,53,54,61,62,63,64,74,85$. All the molars were restored with stainless steel crowns except 84 and 75 . The 84 had to be extracted after injecting $2 \%$ lignocaine adrenaline 1:80,000 and hemostasis was achieved with placement of resorbable sutures in situ. The lower left second molar 75 which was partially erupted was restored with pit and fissure sealant. 51, $52,61,62$ were restored with strip crowns after placement of fibre posts at the coronal one third of the 52,62 to build tooth structure. The upper and lower deciduous canines were restored with light 
cure composite restorations (Figure 2 (c and d)). Preventive protocol was instituted using $2 \%$ sodium fluoride gel in disposable trays applied intraorally. The perioperative period was uneventful, extubation on table performed after removing the throat pack and patient monitored in PACU along with parents and later shifted to ward.

\section{Case Report3}

A 7-year-old male child, a diagnosed case of CP was brought by his parents with the chief complaint of refusal to eat food. On examination it was found that the child had deep dental caries in relation to $65,74,85$. There was a restoration in 75 and 84 was grossly destructed (Figure 3 (a\&b)). The treatment plan was devised for complete rehabilitation: preparatory phase in which the parents were counseled, and dietary instructions were given, a corrective phase which included endodontic treatment and restoration of all restorable teeth and a surgical phase which included extraction of unsavable tooth was drawn out. This treatment plan was discussed with the parents and informed consent was obtained from the parents \& PAC was performed. The child was taken up for full mouth rehabilitation under GA and intubated orally. Pulpectomies were done on $65,74,75,85$ followed by placement of stainless-steel crowns. Grossly destructed 84 was extracted (Figure 3 (c \& d)). Topical fluoride application using $2 \%$ Sodium Fluoride gel was done as a part of the preventive protocol. On completion of the procedure the child was extubated uneventfully and transferred to PACU and later shifted to ward.

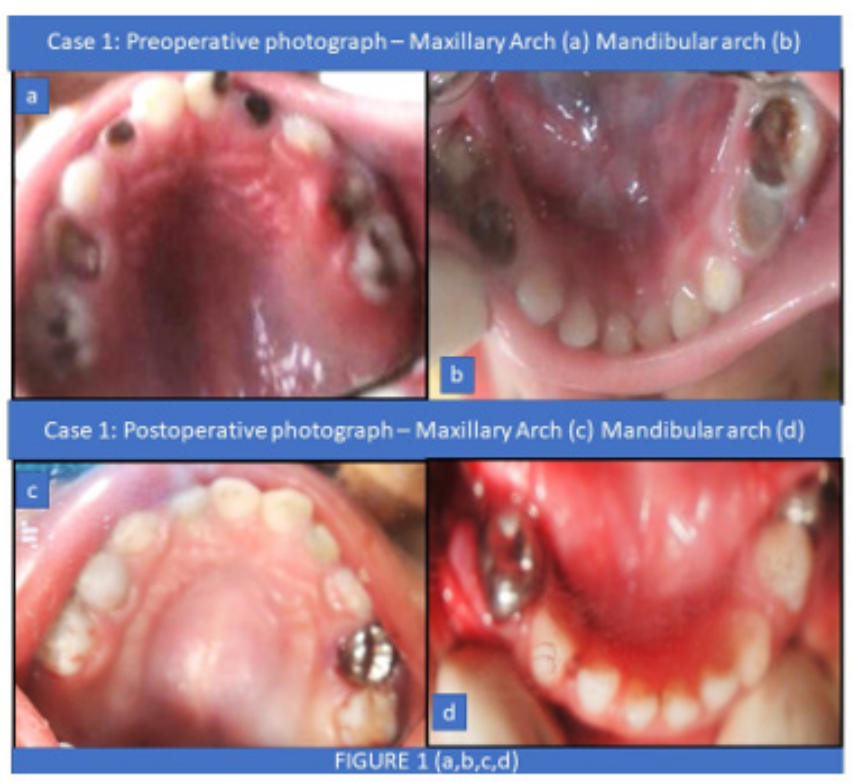

Figure 1 (a, b, c, d): Case 1: Pre-operative and post-operative Maxillary arch (a and b) \& Pre-operative and post-operative Mandibular arch (c and d).

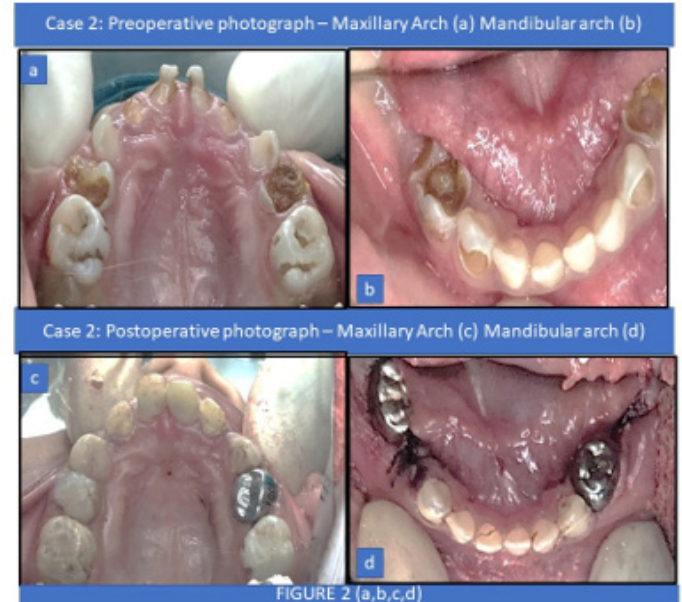

Figure 2 (a, b, c, d): Case 2: Pre-operative and post-operative Maxillary arch (a and b) \& Pre-operative and post-operative Mandibular arch (c and d). 


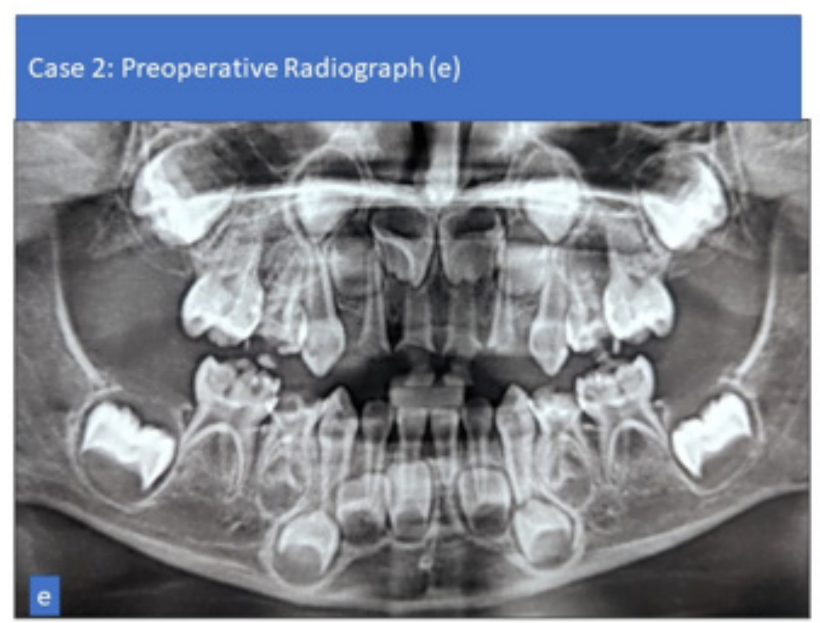

Figure 2e: Preoperative radiograph of case 2.

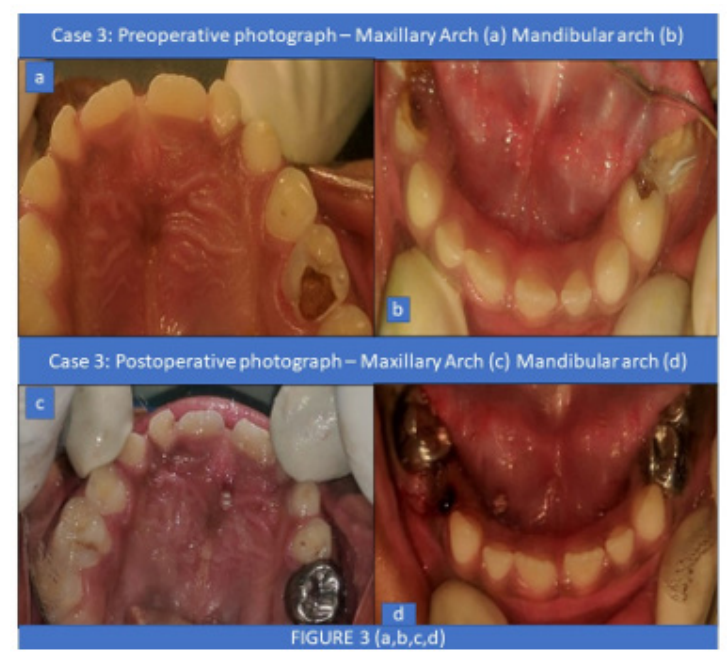

Figure 3 (a, b, c, d): Case 3: Pre-operative and post-operative Maxillary arch (a and c) \& Pre-operative and post-operative Mandibular arch (b and d).

\section{Case Report4}

A 5-year-old male child diagnosed with CP and mental retardation was brought by parents with complaints of pain in oral cavity. The patient was extremely uncooperative and hence a thorough preoperative oral examination was not possible. A treatment plan was devised, which included thorough intra oral examination on operation table after GA and proceeding as per the examination. PAC was carried out and informed consent taken from parents. In the operating room (OR) child was intubated nasally as the oral cavity was very small and oral intubation would have led to difficulty in proper delivery of treatment. The child was examined and found to have generalized hypoplasia (Figure 4 (a \& b)). Pulpectomy was done on $55,65,74,85$. Stainless steel crowns were placed on $54,55,64,65,74$ and 85 with the aim to reduce loss of tooth structure already compromised due to the presence of hypolalsia. Light Cure Composite restorations were done on all the anterior teeth and finally topical fluoride application $2 \%$ sodium fluoride gel was done (Figure 4 (c \& d)). The child was extubated on table and handed over to the parents in PACU and regular follow up routine was instituted.

\section{Case Report5}

A 13-year-old girl child, a diagnosed case of CP, was brought by her parents with complaints of child having discomfort in eating and thus avoided chewing food on the right side. The child made minimal eye contact but understood basic verbal instructions. The child was examined on the dental chair and was found to have root stumps of $15,16,26$. Calculus was present on the lower anterior teeth (Figure 5 (a \& b)). The patient was extremely anxious and uncooperative and hence a thorough preoperative oral examination was not possible. A treatment plan was devised, which included thorough intra oral examination on operation table after GA and proceeding as per the examination. PAC was carried out and informed consent taken from parents. In the OR, patient 
was intubated orally. The preventive protocol in the form of pit and fissure sealant application was done on 14,17, 24, 25, 27. Gross oral prophylaxis was carried out. The root stumps of 15,16,26 was extracted, and hemostasis was achieved (Figure 5 (c \& d)). The child was extubated on table and monitored in PACU.

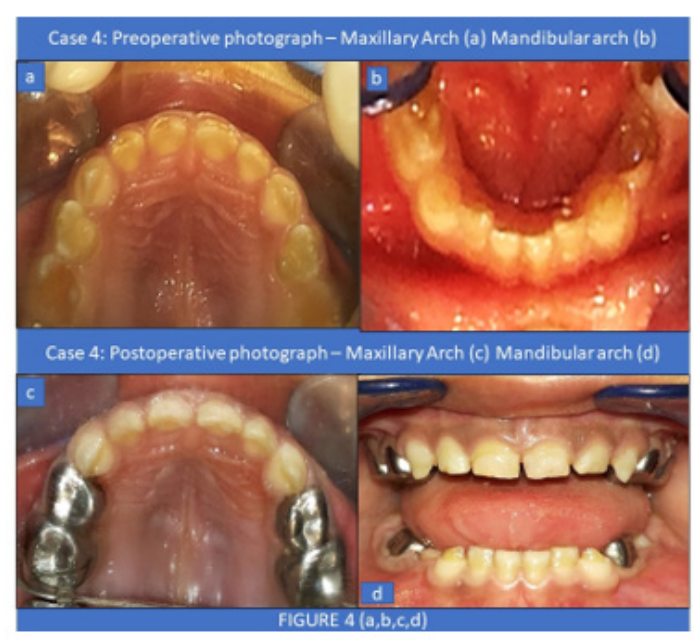

Figure 4 (a, b, c, d): Case 4: Pre-operative and post-operative Maxillary arch (a and c) \& Pre-operative and post-operative Mandibular arch (b and d).

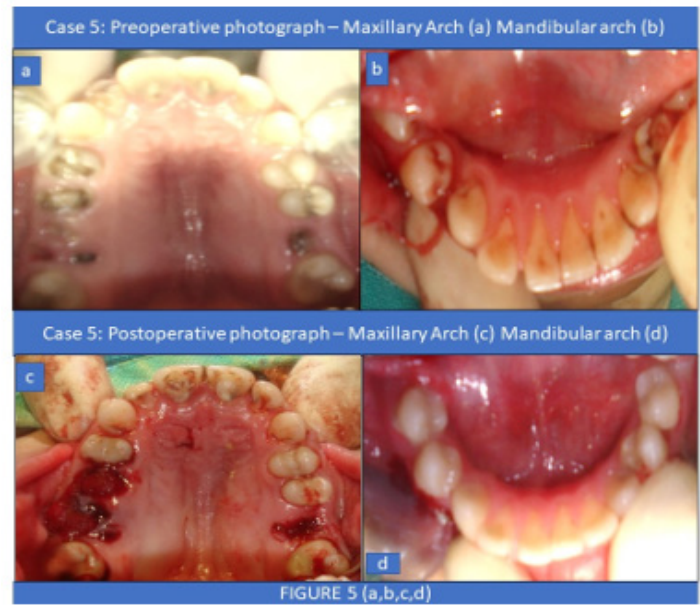

Figure 5 (a, b, c, d): Case 5: Pre-operative and post-operative Maxillary arch (a and c) \& Pre-operative and post-operative Mandibular arch (b and d).

\section{Postoperative instructions and Follow up}

All five children were discharged the following day after the parents were explained about the postoperative care, pain management and recall visits. The children were recalled after a week and the parents were quite satisfied as their child had improved sleep and the dietary pattern had improved considerably. The children were kept on a three month follow up routine.

\section{General Anaesthesia Protocol}

All these patients were diagnosed cases of CP and had some level of mental retardation which made their cooperation elusive and hence required Anaesthetic support not only for the surgical procedure but also for detailed intra-oral assessment. Thus, on chalking out the treatment plan for these children, the parents were counselled extensively to explain the treatment needs and the importance of pharmacological behavioural management due to the children's inability to be able to tolerate the treatment on the dental chair. PAC was carried out, all five children had crepitations in lung bases and airway examination was difficult because of their non-cooperation. Parents were counseled regarding oral and nasal hygiene, respiratory system optimization in form of steam inhalation, nasal douching with normal saline drops and nebulization with normal saline night prior and morning of surgery. Few children were also prescribed antihistaminic. Routine blood and urine examination and tests for viral markers were carried out and a chest X Ray was also performed for all of them. 
Post PAC, children were assigned American Society of Anaesthesiology Physical grading (ASA) II and parents were also counseled regarding perioperative respiratory complications. Informed consent was obtained. Nil per orally status maintained preoperatively and children were taken for full mouth oral rehabilitation under GA. When the children arrived in preoperative room on morning of surgery basal monitoring was attached (Heart rate (HR), pulse oximetry (Sp02) and when the children were transported to OR after premedication the routine monitoring was supplemented (Noninvasive blood pressure (NIBP), electrocardiography (ECG), temperature monitoring along with HR and SpO2). Premedication with intravenous (IV) fentanyl (1microgram $/ \mathrm{kg}$ ) and midazolam $(0.05 \mathrm{mg} / \mathrm{kg})$ was carried out in preoperative room and induced in OR with IV propofol ( $2 \mathrm{mg} / \mathrm{kg}$ ), IV fentanyl (1microgram $/ \mathrm{kg}$ ) and IV vecuronium $(0.1 \mathrm{mg} / \mathrm{kg})$ loading dose for endotracheal intubation. Three children were orally intubated while two children required nasal intubation because their oral cavity was very small and it was difficult for pediatric dental surgeon to work in shared oral space. Endotracheal intubation was carried out with age-appropriate endotracheal tubes (ETT).

Post intubation, capnography and neuromuscular monitor was instituted for monitoring etCO2 and Train of Four to check for muscle relaxation. All children were given throat pack after endotracheal intubation. GA maintained with Air/oxygen/sevoflurane (60:40: Minimum alveolar concentration of 0.8 to 1.0). Intraoperative analgesia was maintained with fentanyl aliquots (0.05 microgram/ $\mathrm{kg}$ ) and paralysis maintained with IV vecuronium $(0.05 \mathrm{mg} / \mathrm{kg})$. Intravenous lines were secured, patient warming system with forced air warming applied to maintain normothermia, paracetamol pediatric suppository given per rectally for perioperative analgesia and patients were handed over for surgical procedure. All the surgeries were uneventful and all the patients could be extubated on table. The surgical time ranged from 180 to 210 minutes while anesthesia time ranged from 220 minutes to 240 minutes. All children were monitored in PACU post-surgery, where they were handed over to parents and after complete recovery shifted to respective wards. Parents were instructed to introduce the child to liquid diet after 6 hours and if well tolerated to commence on semi solid food subsequently.

\section{Discussion}

Cerebral palsy is a central nervous system disorder which affects movement, coordination and posture. It reflects a nonprogressive abnormality due to insult to the immature brain leading to problems related to psychomotor activity along with defects related to sensation, cognition, communication and behavioural problems [11,12]. A variety of factors have been attributed in its aetiology which include (prenatal [35\%], gestational [55\%] or postnatal [10\%]). Several motor areas of the CNS can be affected from the fetal period up to age 5 , with a prevalence of between 2 and 2.5 cases per 1,000 births [13]. The World Health Organization (WHO) estimates that about $10 \%$ of the global population has some form of disability due to different causes and in India, it is $3.8 \%$ of the population of which nearly $15-20 \%$ of the total physically handicapped children suffer from CP [14]. The incidence of CP in India is estimated to be around 3 cases per 1000 live births [15]. Only in 40 to $50 \%$ of the cases the probable cause can be identified [16].

CP can be classified broadly into spastic, dyskinetic ataxic and mixed type. Based on topography it is classified including limbs that are affected, namely diplegia (or diparesis), tri-, tetra-, quadri- or hemiplegia and its severity is described subjectively as mild, moderate or severe [17]. The most common type being the spastic variety accounting for approximately $75 \%$ of the total cases and is primarily due to lesion in cerebrum [18]. The spastic variety is associated with symptoms which include hyperirritability of involved muscles and lack of coordination of intraoral, perioral and masticatory musculature [19]. The dyskinetic type caused by lesion in basal ganglia accounts for approximately $15 \%$ of the cases [20]. Approximately $5 \%$ of the cases are ataxic type and this is caused primarily due to a lesion in cerebellum. The symptoms associated with this type of cerebral palsy are tremors, loss of balance and impaired speech [21]. The mixed variety is seen in approximately $10 \%$ of the cases and is primarily due to a lesion in cerebrum and cerebellum.

The most common mixed cerebral palsy diagnosis is a combination of spastic and athetoid types [22]. This disability usually involves various associated problems such as mental retardation, seizure disorders, sensory deficits, speech and behavior disorders, abnormal joint contractures, periodontal disease, dental caries, malocclusion, bruxism and are prone to trauma [23]. The management of these children poses a challenge for the treating dental surgeon because of uncontrolled involuntary movements, difficulty in communication, inability to open the mouth properly, abnormal posture and multiple dental procedures to be carried out as was seen in the present cases [24]. Hence, GA is resorted to for oral rehabilitation in such children with multiple dental problems [25].

All of our five patients had developed early childhood caries and were in pain and discomfort and required attention to bring about functional normalcy. In three children, it was vital to save their decidous teeth to allow for normal development of the arches. Endodontic therapy to retain these teeth with restoration of functional efficiency was the treatment objective [26]. The primary molars of these children were restored with stainless steel crowns because of the high degree of bruxism seen in these children leading to dental wear [27]. Dietary and home care counselling of the parents prior to discharge along with long term post-operative follow-up is key to successful management of these children [28]. In most instances due to the overall health related issues affecting the child the parents tend to ignore the oral health related issues until pain or discomfort affects the child functionally. 
This is where a multidisciplinary approach helps to not only rehabilitate the child but also achieve long term success in therapeutic and preventive care [29]. The role of the Anaesthesiologist is important in assisting the Pediatric Dentist impart quality rehabilitative care by dealing with the various underlying problems associated with gastrointestinal system, respiratory system, visual and hearing deficits, behavioural issues and epilepsy etc. A thorough Anaesthesia plan needs to be tailored for each patient and adequate care needs to be taken for preparation of the patient for successful and uneventful GA and equal care is required to be delivered for postoperative pain and recovery [30].

\section{Conclusion}

Complete oral rehabilitation of a child with $\mathrm{CP}$ was found to enhance the oral health related quality of life of the child. Guidance and education of caregivers with relation to the special needs of these children along with long term follow-up is the best way to complement therapeutic care under GA for overall well-being of the child. The successful management of these cases depends on smooth interaction between multidisciplines like Peadiatrics, Anaesthesia and Pediatric Oral Surgeon.

\section{Acknowledgement}

None.

\section{Conflict of Interest}

The authors declare no conflict of interest.

\section{References}

1. Bax MGM, Rosebaum P, Leviton A, Paneth N, Dan B (2006) Definition and classification of cerebral palsy. Dev Med Child Neurol 47: 571-576.

2. Jones MW, Morgan E, Shelton JE, Thorogood C (2007) Cerebral palsy: introduction and diagnosis (Part I). J Pediatr Health Care 21(3): 146152.

3. Wass CT, Warner ME, Worrell GA, Julie A Castagno, Melinda Howe, et al. (2012) Effect of general anesthesia in patients with cerebral palsy at the turn of the new millennium: a population-based study evaluating perioperative outcome and brief overview of anesthetic implications of this coexisting disease. J Child Neurol 27(7): 859-866.

4. Sehrawat N, Marwaha M, Kalpana B, Radhika C (2014) Cerebral Palsy: A Dental Update. Int J Clin Pediatr Dent 7: 109-118.

5. Al-Allaq T, DeBord TK, Liu H, Wang Y, Messadi DV (2015) Oral health status of individuals with cerebral palsy at a nationally recognized rehabilitation center. Spec Care Dentist 35(1): 15-21.

6. Rodrigues dos Santos MT, Masiero D, Novo NF, Simionato MR (2003) Oral conditions in children with cerebral palsy. J Dent Child (Chic) 70: 40-46.

7. Winter K, Baccaglini L, Tomar S (2008) A review of malocclusion among individuals with mental and physical disabilities. Spec Care Dentist 28 19-25.

8. Jabarifar SE, Eshghi AR, Shabanian M (2009) Changes in children's oral health related quality of life following dental treatment under general anesthesia. Dent Res J (Isfahan) 6(1): 13-16.

9. Bello LL (2000) A Retrospective study of pediatric dental patients treated under general anesthesia. Saudi Dent J 12: 10-15.
10. Theroux MC, Akins RE (2005) Surgery and anesthesia for children who have cerebral palsy. Anesthesiol Clin North America 23: 733-743.

11. Silvestre F, Martinez V (2001) Maneuver odontologico del paciente con parlisiscerebral. Manual maneuver odontolgico el paciente discapacitado psíquico; Valencia, Madrid: Procter \& Gamble SA, pp: 41-48.

12. Alejandro Escanilla C, Mirella Aznar G, Jose Maria V, Ana Lopez G, Alejandro Rivera B (2014) Dental treatment under general anesthesia in a group of patients with cerebral palsy and a group of healthy pediatric patients. Med Oral Patol Oral Cir Bucal 19(5): e490-494.

13. Mutch LW, Alberman E, Hagberg B, Kodama K, Velickovic MV (1992) Cerebral palsy epidemiology: where are we now and where are we going? Dev Med Child Neurol 34: 547-555.

14. Vyas AG, Kori VK, Rajagopala S, Patel KS (2013) Etiopathological study on cerebral palsy and its management by Shashtika Shali Pinda Sweda and Samvardhana Ghrita. Ayu 34(1): 56-62.

15. Kathy Jones, c1997-2013, Medindia.net, MedIndia Inc, Incidence of Cerebral Palsy Remains Constant in India on Indian Health News.

16. Parthasarathy A (2009) ( $4^{\text {th }}$ edn.) Jaypee Digital IAP Textbook of Pediatrics 2: 1045.

17. Graham HK (2005) Classifying cerebral palsy. J Pediatr Orthop 25: 127 128.

18. Paulson A, Vargus Adams J (2017) Overview of Four Functional Classification Systems Commonly Used in Cerebral Palsy. Children (Basel) 4(4): 30.

19. Shamsoddini A, Amirsalari S, Hollisaz MT, Rahimnia A, Khatibi Aghda A (2014) Management of spasticity in children with cerebral palsy. Iran J Pediatr 24(4): 345-351.

20. Monbaliu E, Himmelmann K, Lin JP, Ortibus E, Bonouvrié L, et al. (2017) Clinical presentation and management of dyskinetic cerebral palsy. Lancet Neurol 16(9): 741-749.

21. Bernard D (2020) How useful is the diagnosis of ataxic cerebral palsy? Developmental Medicine and Child Neurology 62(3):261- 264

22. Hagberg B, Sanner G, Steen M (1972) The disequilibrium syndrome in cerebral palsy: clinical aspects and treatment. Acta Paediatr Scand 61(suppl 226): 1-63.

23. Kumar S, Sharma J, Duraiswamy P, Kulkarni S (2009) Determinants for oral hygiene and periodontal status among mentally disabled children and adolescents. J Ind Soc Pedod Prev Dent 27(3): 151-157.

24. Sherawat N, Marwaha M, Bansal K, Chopra R (2014) Cerebral palsy a dental update. Int J Clin Pediatr Dent 7(2): 109-118.

25. Cantekin K, Dogan S, Aydinbelge M, Canpolat DG, Yildirim MD (2014) Analysis of comprehensive dental rehabilitation under general anesthesia at a dental hospital in Turkey. JOPD 2(2): 49-55.

26. Santos MT, Guare RO, Celiberti P, Siqueira WL (2009) Caries experience in individuals with cerebral palsy in relation to oromotor dys- function and dietary consistency. Spec Care Dentist 29(5): 198-203.

27. Cristiana Aroeira Oliveira GR, Viviane Andrade Cancio de Paula, Maristela Barbosa Portela, Laura Salignac Guimaraes, Gloria Fernando Castro (2011) Bruxism control in child with cerebral palsy. ISRN Dent.

28. Mohan DU, Beena JP, Divya R (2010) Importance of oral hygiene habits in mentally disabled children. Int J Clin Pediatr Dent 3(1): 39-42.

29. Jaya AR, Praveen P, Ananthraj A, Nidhi K Nihal (2014) Full mouth rehabilitation of a child with cerebral palsy under general anaesthesia. J Health Sci Res 5(2): 29-32.

30. Nolan J, Chalkiadis GA, Low J, Olesch CA, Brown TCK (2002) Anaesthesia and pain management in cerebral palsy. Anaesthesia 55: 32-41. 